\title{
Coherent spin mixing dynamics in a spin-1 atomic condensate
}

\author{
Wenxian Zhang ${ }^{1}$, D. L. Zhou ${ }^{1,2}$, M. -S. Chang ${ }^{1}$, M. S. Chapman ${ }^{1}$, and L. You ${ }^{1,2}$ \\ ${ }^{1}$ School of Physics, Georgia Institute of Technology, Atlanta, GA 30332-0430 and \\ ${ }^{2}$ Institute of Theoretical Physics, The Chinese Academy of Sciences, Beijing 100080, China
}

(Dated: Submitted on August 13, 2004)

\begin{abstract}
We study the coherent off-equilibrium spin mixing inside an atomic condensate. Using mean field theory and adopting the single spatial mode approximation (SMA), the condensate spin dynamics is found to be well described by that of a nonrigid pendulum, and displays a variety of periodic oscillations in an external magnetic field. Our results illuminate several recent experimental observations and provide critical insights into the observation of coherent interaction-driven oscillations in a spin-1 condensate.
\end{abstract}

PACS numbers: 03.75.Mn, 03.75.Kk

Keywords: Spin-1 BEC, Spin mixing dynamics, Zeeman effect

Bose-Einstein condensation (BEC) has been one of the most active topics in physics for over a decade, and yet interest in this field remains impressively high. Recent experiments showcase the rich versatility of control over the atomic superfluid, e.g. the BEC-BCS crossover 1, 2], quantized vortices 3, 4, 5], condensates in optical lattices [6], and low dimensional quantum gases [7, 8]. While most of these efforts involve condensates of atoms in a single Zeeman state, activities in spinor condensates [9, 10] have recently received significant boost with the addition of three new spin-1 experiments [1, 12, 13, 14].

In a spinor condensate, atomic hyperfine spin degree of freedom becomes accessible with the use of a far-off resonant optical trap instead of a magnetic trap. For atoms in the $F=1$ ground state manifold, the presence of Zeeman degeneracy and spin dependent atom-atom interactions [9, 10, 11, 15, 16, 17, 18, 19] leads to interesting condensate spin dynamics. In this article, we study spin mixing inside a spin- 1 condensate [17, 19, 20], focusing on the interaction-driven coherent oscillations within a mean field description. Unlike the pioneering studies on this subject as in Refs. 17, 19], we will highlight the important role of an external magnetic field, which is present in all experiments to date.

Recently, a beautiful experiment has finally observed the long predicted Josephson type coherent nonlinear oscillations with a scalar condensate in a spatial double well potential [21].

Although spin mixing driven by the internal spindependent interaction (not of the nature of a Rabi oscillation as driven by an external field [22, 23]), has been observed in both $F=1$ and $F=2$ condensates [9, 12, 14, 24], the coherence of this process has not yet been investigated. Over-damped single oscillations in spin populations have been observed in earlier experiments 24] although their interpretation has been limited because evolution from the initial (meta-stable) states was noise-driven. The main experimental obstacles to observe more oscillations are the dissipative atomic collisions among the condensed atoms and the decoherence collisions with noncondensed atoms 12, 24]. A promising future direction relies on increased atomic detection

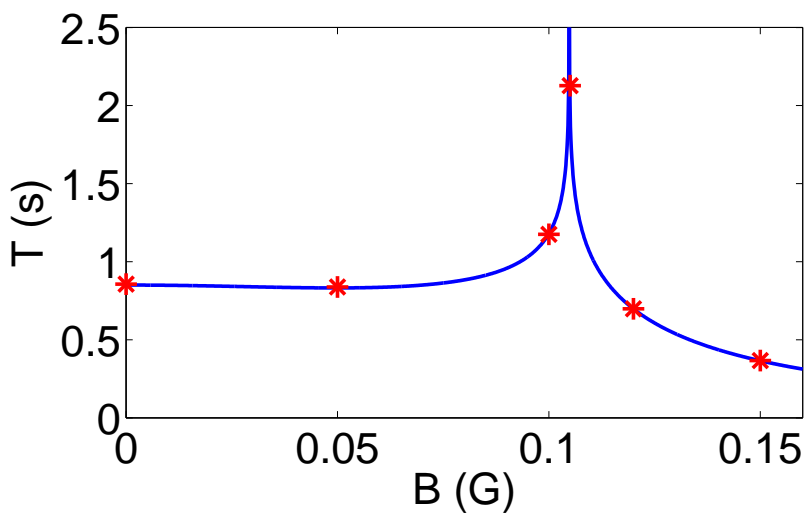

FIG. 1: (Color online) The dependence of oscillation period $T$ on magnetic field $B$ for a ${ }^{87} \mathrm{Rb}$ condensate from our model (solid line, Eq. (8a)), the results from a full numerical simulation without the use of SMA are denoted by $\left(^{*}\right)$.

sensitivity, thus the use of smaller condensates as in the experiment of Ref. [21], with lower number densities and at lower temperatures, two favorable conditions for the single spatial mode approximation (SMA) [17, 19].

The initial atomic population distribution in Fig. 1 corresponds to the (equilibrium) ground state at a magnetic field (B-field) of 0.07 Gauss and with a zero magnetization $(m=0)$, specified by $\rho_{0}(0) \approx 0.644$ and $\theta(0)=0$ with $c \approx 0.614 \mathrm{~Hz}$ (these symbols are defined later). As in the case of no B-fields [17, 18, 19], the initial relative phases among the three components depend on the spindependent atom-atom interaction being ferromagnetic $(0)$ or antiferromagnetic $(\pi)$, inside an external magnetic field 25]. Starting with the initial phases and population distributions at $B_{0}=0.07$ Gauss, we instantaneously change the $B$-field to a different value, the atomic condensate distributions thus become off-equilibrium, and the coherent dynamics starts according to the mean field theory. Within the SMA, we find such off-equilibrium dynamics of a spin- 1 condensate corresponds to that of a nonrigid pendulum, which can be characterized using semiclassical trajectories in the phase space. 
Our main result is illustrated in Fig. 1 where we have plotted the dependence of oscillation period on the external magnetic field. The parameters are close to the experiment [26]; where the spin-independent trap is harmonic $V=(M / 2)\left(\omega_{x}^{2} x^{2}+\omega_{y}^{2} y^{2}+\omega_{z}^{2} z^{2}\right)$ with $\omega_{x}=\omega_{y}=$ $(2 \pi) 240 \mathrm{~Hz}$ and $\omega_{z}=(2 \pi) 24 \mathrm{~Hz}$. The condensate contains $N=1,000{ }^{87} \mathrm{Rb}$ atoms with an average density of $\langle n\rangle \approx 1.7 \times 10^{13} \mathrm{~cm}^{-3}$.

As illustrated in Fig. 11 the spin mixing dynamics within the SMA corresponds to a typical pendulum, with the quadratic Zeeman energy playing the important role of the total energy. At small change of $B$-field, the equivalent pendulum undergoes a small amplitude oscillation, approximately harmonic with a period independent of the energy or oscillation amplitude; increasing of the total energy leads to a longer oscillation period as the pendulum becomes increasingly nonlinear. At a critical field $B_{c}$, when the effective total energy is just enough to bring the pendulum to the completely up or top position, the period approaches infinity as for the homoclinic orbit of a pendulum; Upon further increasing the energy (or $B$ ), the pendulum starts to rotate around and the period becomes smaller with increasing energy as the pendulum rotates faster and faster.

Our system of a spin- 1 atomic Bose gas inside an external magnetic field is described by the Hamiltonian [15, 16]

$$
\begin{aligned}
\mathcal{H}= & \int d \mathbf{r}\left[\psi_{i}^{\dagger}\left(-\frac{\hbar^{2}}{2 M} \nabla^{2}+V+E_{i}\right) \psi_{i}+\frac{c_{0}}{2} \psi_{i}^{\dagger} \psi_{j}^{\dagger} \psi_{j} \psi_{i}\right. \\
& \left.+\frac{c_{2}}{2} \psi_{k}^{\dagger} \psi_{i}^{\dagger}\left(F_{\gamma}\right)_{i j}\left(F_{\gamma}\right)_{k l} \psi_{j} \psi_{l}\right],
\end{aligned}
$$

where repeated indices are summed, and $\psi_{i}(\mathbf{r})\left(\psi_{i}^{\dagger}\right)$ is the field operator that annihilates (creates) an atom in the $i$-th hyperfine state $(|F=1, i=+1,0,-1\rangle$, hereafter $|i\rangle)$ at location $\mathbf{r}$. $M$ is the mass of an atom. Interaction terms with coefficients $c_{0}$ and $c_{2}$ describe respectively elastic collisions of spin- 1 atoms, expressed in terms of the scattering length $a_{0}\left(a_{2}\right)$ for two spin-1 atoms in the combined symmetric channel of total spin $0(2)$, $c_{0}=4 \pi \hbar^{2}\left(a_{0}+2 a_{2}\right) / 3 M$ and $c_{2}=4 \pi \hbar^{2}\left(a_{2}-a_{0}\right) / 3 M$. $F_{\gamma=x, y, z}$ are spin- 1 matrices. Assuming the external magnetic field $\mathbf{B}$ to be along the quantization axis $(\hat{z})$, the Zeeman shift on an atom in state $|i\rangle$ becomes (the BreitRabi formula 27])

$$
\begin{aligned}
& E_{ \pm}=-\frac{E_{\mathrm{HFS}}}{8} \mp g_{I} \mu_{I} B-\frac{1}{2} E_{\mathrm{HFS}} \sqrt{1 \pm \alpha+\alpha^{2}}, \\
& E_{0}=-\frac{E_{\mathrm{HFS}}}{8}-\frac{1}{2} E_{\mathrm{HFS}} \sqrt{1+\alpha^{2}},
\end{aligned}
$$

where $E_{\mathrm{HFS}}$ is hyperfine splitting, and $g_{I}$ is the Lande $g$-factor for an atom with nuclear spin $\mathbf{I}$. $\mu_{I}$ is the nuclear magneton and $\alpha=\left(g_{I} \mu_{I} B+g_{J} \mu_{B} B\right) / E_{\mathrm{HFS}}$ with $g_{J}$ representing Lande $g$-factor for a valence electron with a total angular momentum $\mathbf{J} . \mu_{B}$ is the Bohr magneton.

The field operators $\psi_{i}$ evolve according to the Heisenberg operator equation of motion. At near-zero temperature and when the total number of condensed atoms
$(N)$ is large, the condensate is essentially described by the mean field $\phi_{i}=\left\langle\psi_{i}\right\rangle$. Neglecting quantum fluctuations, they form a set of coupled Gross-Pitaevskii (GP) equations, from which we can simulate the mean field off-equilibrium dynamics more accurately at various external magnetic fields without using the SMA.

Our simplified model is based on the well-known fact that for both ${ }^{87} \mathrm{Rb}$ (ferromagnetic) and ${ }^{23} \mathrm{Na}$ (antiferromagnetic) atoms, the spin dependent interaction $\propto\left|c_{2}\right|$ is much weaker than the density dependent interaction $\propto\left|c_{0}\right|$. This leads to the validity of the SMA, where we adopt the mode function $\phi(\mathbf{r})$ as determined from the spin-independent part of the Hamiltonian $\mathcal{H}_{s}=$ $-\left(\hbar^{2} / 2 M\right) \nabla^{2}+V+c_{0} n[17,18,19]$. Thus we define

$$
\phi_{i}(\mathbf{r}, t)=\sqrt{N} \xi_{i}(t) \phi(\mathbf{r}) \exp (-i \mu t / \hbar),
$$

where $\mathcal{H}_{s} \phi(\mathbf{r})=\mu \phi(\mathbf{r})$ and $\int d \mathbf{r}|\phi(\mathbf{r})|^{2}=1$. We arrive at the coupled spinor equations

$$
\begin{aligned}
i \hbar \dot{\xi_{ \pm}} & =E_{ \pm} \xi_{ \pm}+c\left[\left(\rho_{ \pm}+\rho_{0}-\rho_{\mp}\right) \xi_{ \pm}+\xi_{0}^{2} \xi_{\mp}^{*}\right], \\
i \hbar \dot{\xi_{0}} & =E_{0} \xi_{0}+c\left[\left(\rho_{+}+\rho_{-}\right) \xi_{0}+2 \xi_{+} \xi_{-} \xi_{0}^{*}\right],
\end{aligned}
$$

with $c=c_{2} N \int d \mathbf{r}|\phi(\mathbf{r})|^{4}, \rho_{i}=\left|\xi_{i}\right|^{2}$. It is easy to verify that the total atom number and atomic magnetization are conserved, i.e. $\sum_{i} \rho_{i} \equiv 1, \rho_{+}-\rho_{-} \equiv m$, and $m=$ $\left(N_{+}-N_{-}\right) / N$ is a constant of motion.

We use $\eta=\left(E_{-}-E_{+}\right) / 2$ and $\delta=\left(E_{-}+E_{+}-2 E_{0}\right) / 2$ to parameterize the linear and quadratic Zeeman effect. We further transform

$$
\begin{aligned}
\xi_{+} & \rightarrow \xi_{+} \exp \left[-i\left(E_{0}-\eta\right) t / \hbar\right] \\
\xi_{0} & \rightarrow \xi_{0} \exp \left[-i E_{0} t / \hbar\right] \\
\xi_{-} & \rightarrow \xi_{-} \exp \left[-i\left(E_{0}+\eta\right) t / \hbar\right]
\end{aligned}
$$

to eliminate the $E_{0}$ and $\eta$ dependence, and take $\xi_{j}=$ $\sqrt{\rho_{j}} e^{-i \theta_{j}}$. After some simplification, we obtain the following dynamic equations for spin mixing inside a spin-1 condensate

$$
\begin{aligned}
\dot{\rho}_{0} & =\frac{2 c}{\hbar} \rho_{0} \sqrt{\left(1-\rho_{0}\right)^{2}-m^{2}} \sin \theta, \\
\dot{\theta} & =-\frac{2 \delta}{\hbar}+\frac{2 c}{\hbar}\left(1-2 \rho_{0}\right) \\
& +\left(\frac{2 c}{\hbar}\right) \frac{\left(1-\rho_{0}\right)\left(1-2 \rho_{0}\right)-m^{2}}{\sqrt{\left(1-\rho_{0}\right)^{2}-m^{2}}} \cos \theta,
\end{aligned}
$$

where $\theta=\theta_{+}+\theta_{-}-2 \theta_{0}$ is the relative phase. These two coupled equations give rise to a classical dynamics of a nonrigid pendulum, whose energy functional (or Hamiltonian) can also be derived within the SMA as in 18]

$$
\begin{aligned}
\mathcal{E}= & c \rho_{0}\left[\left(1-\rho_{0}\right)+\sqrt{\left(1-\rho_{0}\right)^{2}-m^{2}} \cos \theta\right] \\
& +\delta\left(1-\rho_{0}\right) .
\end{aligned}
$$

It is easy to check that $\dot{\rho}_{0}=-(2 / \hbar) \partial \mathcal{E} / \partial \theta$ and $\dot{\theta}=$ $(2 / \hbar) \partial \mathcal{E} / \partial \rho_{0}$. 


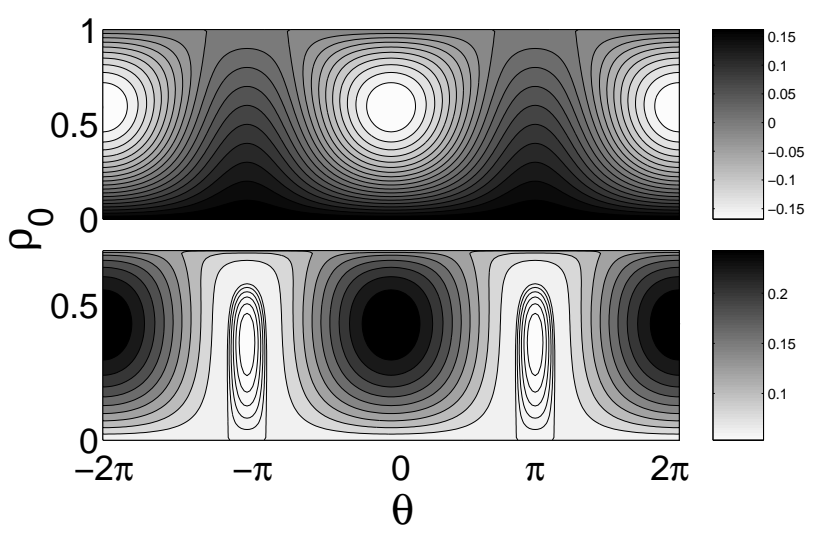

FIG. 2: Equal-energy contours for a condensate of ${ }^{87} \mathrm{Rb}$ atoms (upper panel) with $B=0.05$ Gauss, $|c|=(2 \pi) 0.5 \mathrm{~Hz}$, and $m=0$; of ${ }^{23} \mathrm{Na}$ atoms (lower panel) with $B=0.015$ Gauss, $|c|=(2 \pi) 0.5 \mathrm{~Hz}$, and $m=0.3$.

The contour plot of $\mathcal{E}$ in Fig. 2 displays several types of oscillation as in a pendulum. The dynamics of spin mixing described by Eqs. (4. 5) in a magnetic field is conservative, as also recognized and studied numerically in Ref. 28]. The corresponding phase space trajectory is therefore confined to stay on the equal energy contour. Quite generally, $\rho_{0}$ oscillates in a magnetic field. Rewriting equation (4) as

$$
\begin{gathered}
\left(\dot{\rho}_{0}\right)^{2}=\frac{4}{\hbar^{2}}\left\{\left[\mathcal{E}-\delta\left(1-\rho_{0}\right)\right]\left[\left(2 c \rho_{0}+\delta\right)\left(1-\rho_{0}\right)-\mathcal{E}\right]\right. \\
\left.-\left(c \rho_{0} m\right)^{2}\right\}
\end{gathered}
$$

we can compute the oscillation period according to

$$
T=\oint \frac{1}{\dot{\rho}_{0}} d \rho_{0}=\frac{\sqrt{2} \hbar}{\sqrt{-\delta c}} \frac{K\left(\sqrt{\frac{x_{2}-x_{1}}{x_{3}-x_{1}}}\right)}{\sqrt{x_{3}-x_{1}}}, \text { for } c<0,
$$

and

$$
T=\frac{\sqrt{2} \hbar}{\sqrt{\delta c}} \frac{K\left(\sqrt{\frac{x_{3}-x_{2}}{x_{3}-x_{1}}}\right)}{\sqrt{x_{3}-x_{1}}}, \text { for } c>0 .
$$

$K(k)$ is the elliptic integral of the first kind, and $x_{j=1,2,3}$ are the roots of $\dot{\rho}_{0}=0$ (order as $x_{1} \leq$ $\left.x_{2} \leq x_{3}\right)$ (Fig. 3). The period for a rigid pendulum, described by $\ddot{u}+\sin u=0$, is $T=$ $4 \sqrt{2} K[\sqrt{2 /(E+1)}] / \sqrt{E+1}$ at an energy $E>1$ and $T=4 \sqrt{2} F[\arcsin (\sqrt{(E+1) / 2}), \sqrt{2 /(E+1)}] / \sqrt{E+1}$ when $-1 \leq E \leq 1$. Here $E$ is the energy of the rigid pendulum.

The time evolution of $\rho_{0}$ can be expressed in terms of the Jacobian elliptic function $\mathrm{cn}(.,$.$) ,$

$$
\begin{aligned}
\rho_{0}(t)= & x_{2}-\left(x_{2}-x_{1}\right) \mathrm{cn}^{2}\left[\gamma_{0}+t \sqrt{-2 \delta c\left(x_{3}-x_{1}\right)}, k\right], \\
& \text { for } c<0,
\end{aligned}
$$

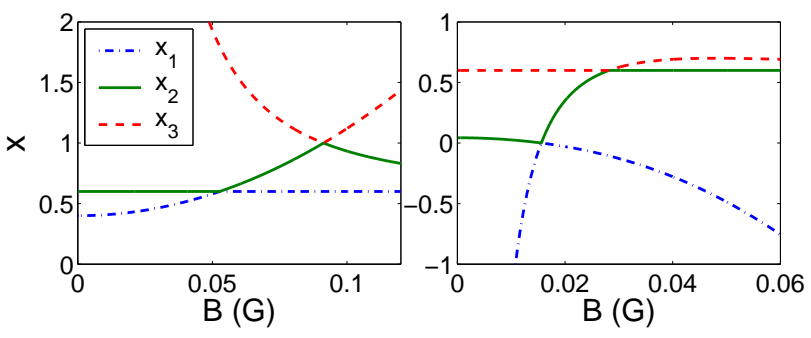

FIG. 3: (Color online) The dependence of cubic roots $x_{j}$ on the external magnetic field for ${ }^{87} \mathrm{Rb}$ atoms (left) and ${ }^{23} \mathrm{Na}$ atoms (right). Other parameters are $|c|=(2 \pi) 0.5 \mathrm{~Hz}, \rho_{0}(0)=$ $0.6, \theta(0)=0$, and $m=0$ for ${ }^{87} \mathrm{Rb} ; \theta(0)=\pi$ and $m=0.3$ for ${ }^{23} \mathrm{Na}$.
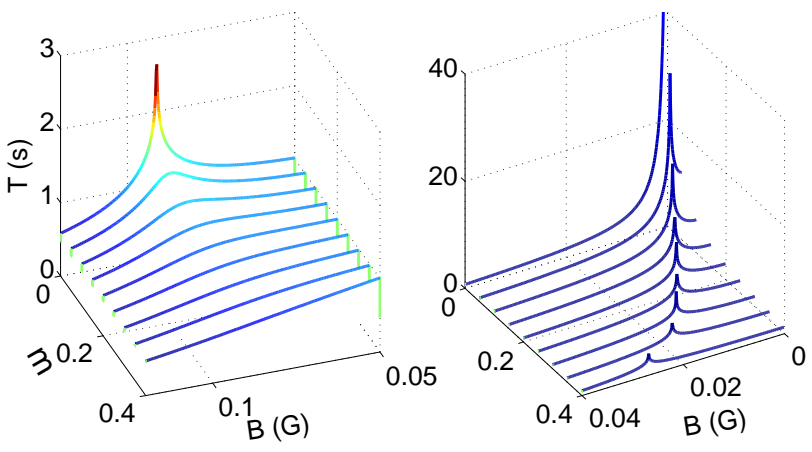

FIG. 4: (Color online) The magnetic field dependence of the oscillation period for ${ }^{87} \mathrm{Rb}$ atoms (left) and ${ }^{23} \mathrm{Na}$ atoms (right). Other parameters are the same as in Fig. 3

and

$$
\begin{aligned}
\rho_{0}(t)= & x_{3}-\left(x_{3}-x_{2}\right) \mathrm{cn}^{2}\left[\gamma_{0}+t \sqrt{2 \delta c\left(x_{3}-x_{1}\right)}, k\right], \\
& \text { for } c>0,
\end{aligned}
$$

$\gamma_{0}$ depends on the initial state, $\operatorname{cn}^{2}\left(\gamma_{0}, k\right)=\left[x_{2}-\right.$ $\left.\rho_{0}(0)\right] /\left(x_{2}-x_{1}\right)$ if $c<0$ and $\operatorname{cn}^{2}\left(\gamma_{0}, k\right)=\left[x_{3}-\right.$ $\left.\rho_{0}(0)\right] /\left(x_{3}-x_{2}\right)$ if $c>0$. For ${ }^{87} \mathrm{Rb}$ atoms $(c<0), \gamma_{0}=0$ if $\rho_{0}(0)=x_{1}$ and $\gamma_{0}=K(k)$ if $\rho_{0}(0)=x_{2}$. The solutions of $\rho_{0}$ are oscillatory between $x_{1}$ and $x_{2}$ if $c<0$ (between $x_{2}$ and $x_{3}$ if $\left.c>0\right)$, except when $x_{2}=x_{3}\left(x_{2}=x_{1}\right.$ if $c>0$ ), where the solution becomes homoclinic, i.e., $\lim _{t \rightarrow \infty} \rho_{0}=1$ and the corresponding period is infinity for $m=0$ (Fig. 41).

We further observe from Fig. [4 that when the total magnetization is varied the peak of the oscillation period essentially stays at the same magnetic field for ferromagnetic interactions. The solution becomes periodic when $m \neq 0$ since $\rho_{0}$ can at most reach $1-m$. It turns out that the critical solution of an infinitely long oscillation period occurs when $\rho_{0}(t \rightarrow \infty)=1$, or equivalently $\mathcal{E}=0$, which gives $\delta\left(B_{c}\right)=|c| \rho_{0}(1+\cos \theta)$ with $\rho_{0}$ and $\theta$ the initial conditions. At $B=0$ we reproduce the same result as in Ref. [19]. The rapid decreasing of the period when $B>B_{c}$ is consistent with the recent numerical simulations by Schmaljohann et al. 13]. For antiferromagnetic interactions, however, the peak of the oscillation period shows 
a strong dependence on the magnetization, and asymptotically we find $\rho_{0}(t \rightarrow \infty)=0$, i.e., $\mathcal{E}=\delta\left(B_{c}\right)$ which is equivalent to $\delta\left(B_{c}\right)=c\left[\left(1-\rho_{0}\right)+\sqrt{\left(1-\rho_{0}\right)^{2}-m^{2}} \cos \theta\right]$.

Substituting the solution $\rho_{0}(t)$ into Eq. (6), we can solve for $\theta(t)$. Furthermore we can find the time dependence of $\theta_{ \pm}$and $\theta_{0}$ through the following

$$
\begin{aligned}
& \dot{\theta}_{ \pm}=-\frac{1}{\hbar}\left[\delta+c \rho_{0}+c \rho_{0} \sqrt{\frac{1-\rho_{0} \mp m}{1-\rho_{0} \pm m}} \cos \theta\right] \\
& \dot{\theta}_{0}=-\frac{c}{\hbar}\left[\left(1-\rho_{0}\right)+\sqrt{\left(1-\rho_{0}\right)^{2}-m^{2}} \cos \theta\right] .
\end{aligned}
$$

Finally, we consider the evolution of the averaged total spin. As was recently demonstrated by Higbie et al., the averaged spin of a condensate or its magnetization can be directly probed with non-destructive phase contrast imaging [29]. Alternatively, the magnetization dynamics can be inferred from component populations of a spinor condensate, which are directly measurable using SternGerlach effect in an inhomogeneous magnetic field. We first illustrate the quadratic Zeeman effect on the spin dynamics of a noninteracting condensate. For a state $\xi=\left(\xi_{+}, \xi_{0}, \xi_{-}\right)^{T}$, the total spin average is $\langle\mathbf{F}\rangle=\langle\xi| F_{x} \hat{x}+$ $F_{y} \hat{y}+F_{z} \hat{z}|\xi\rangle$ with

$$
\begin{aligned}
\left\langle F_{x}\right\rangle & =\sqrt{2} \operatorname{Re}\left[\left|\xi_{0}\right|\left(\left|\xi_{+}\right| e^{i\left(\theta_{0}-\theta_{+}\right)}+\left|\xi_{-}\right| e^{i\left(\theta_{0}-\theta_{-}\right)}\right)\right], \\
\left\langle F_{y}\right\rangle & =\sqrt{2} \operatorname{Im}\left[\left|\xi_{0}\right|\left(\left|\xi_{+}\right| e^{i\left(\theta_{0}-\theta_{+}\right)}-\left|\xi_{-}\right| e^{i\left(\theta_{0}-\theta_{-}\right)}\right)\right], \\
\left\langle F_{z}\right\rangle & =\left|\xi_{+}\right|^{2}-\left|\xi_{-}\right|^{2}=m .
\end{aligned}
$$

As an interesting case, we take the initial state as $\xi(0)=$ $\left[\sqrt{\left(1-\rho_{0}\right) / 2}, \sqrt{\rho_{0}}, \sqrt{\left(1-\rho_{0}\right) / 2}\right]^{T} \cdot \rho_{0}$ is a constant. We find at time $t$ that

$$
\begin{aligned}
\left\langle F_{x}\right\rangle+i\left\langle F_{y}\right\rangle & =2 \sqrt{\rho_{0}\left(1-\rho_{0}\right)} \cos (\delta t / \hbar) e^{-i \eta t / \hbar} \\
\left\langle F_{z}\right\rangle & =m=0
\end{aligned}
$$

It spirals toward and away from the origin in the $\left\langle F_{x}\right\rangle$ $\left\langle F_{y}\right\rangle$ plane. The linear Zeeman effect causes spin precessing around the magnetic field ( $\hat{z}$ axis), while the quadratic Zeeman effect makes spin average oscillate.

The spin evolution becomes quite different when atom interaction is present. For the same initial conditions (of the above), the total averaged spin at time $t$ becomes

$$
\begin{aligned}
\left\langle F_{x}\right\rangle+i\left\langle F_{y}\right\rangle & =2 \sqrt{\rho_{0}\left(1-\rho_{0}\right)} \cos (\theta / 2) e^{-i \eta t / \hbar} \\
\left\langle F_{z}\right\rangle & =0
\end{aligned}
$$

which can be conveniently confirmed from the phase space contour plot of Fig. [2] where $\theta$ is confined to oscillate around zero for ferromagnetic interactions and around $\pi$ for antiferromagnetic interactions if $B<B_{c}$. Note that $\rho_{0}$ and $\theta$ are time-dependent for interacting condensates. Figure 5 exemplifies this oscillation in terms of the allowed regions (shaded) of $\left\langle F_{x}\right\rangle$ and $\left\langle F_{y}\right\rangle$ for interacting condensates in contrast to noninteracting ones. For ferromagnetic interactions, the

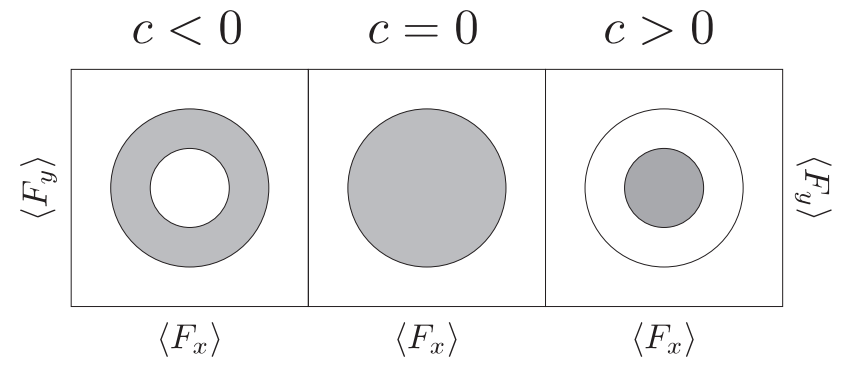

FIG. 5: Two dimensional projection of the averaged spin evolution (shaded region) for a condensate with zero magnetization of noninteracting atoms (middle), in comparison with atoms of ferromagnetic (left) and antiferromagnetic interactions (right).

allowed region is defined by two radii. One of them, $r_{I}=\sqrt{2 \rho_{0}(0)\left[\left(1-\rho_{0}(0)\right)+\sqrt{\left(1-\rho_{0}(0)\right)^{2}-m^{2}}\right]}$, depends on the initial condition, while the other $\left(r_{B}\right)$ is solely determined by the quadratic Zeeman effect. We find $r_{B}>r_{I}$ if $B<B_{0}, 0<r_{B}<r_{I}$ if $B_{0}<B<B_{c}$, and $r_{B}=0$ if $B \geq B_{c}$. There exists a forbidden region at the center for a ferromagnetically interacting condensate if $B<B_{c}$. This region shrinks to zero when $B \geq B_{c}$. Exactly at $B=B_{c}$, an interesting attractor-like feature arises and the average spin gradually spirals towards the origin (at the center) and becomes trapped eventually after an infinitely long time. For antiferromagnetic interactions, the allowed region generally becomes smaller than that for a noninteracting condensate as shown in Fig. 5 for $m=0$ (or $B_{c}=0$ ). The radius of the shaded (allowed) region depends on the quadratic Zeeman effect, while the forbidden region approaches zero as $B \rightarrow \infty$.

For the general case of $m \neq 0$, the allowed region is in between the two radii $\sqrt{2 \rho_{0}(0)\left[\left(1-\rho_{0}(0)\right) \pm \sqrt{\left(1-\rho_{0}(0)\right)^{2}-m^{2}}\right]}$ for a noninteracting gas, For ferromagnetic interactions, the averaged spin behaves similar to the case of $m=0$ considered above, except now the forbidden region shrinks gradually to a minimum nonzero value of $\sqrt{2 \rho_{0}(0)\left[\left(1-\rho_{0}(0)\right)-\sqrt{\left(1-\rho_{0}(0)\right)^{2}-m^{2}}\right.}$ when $B \rightarrow \infty$. In this case, there exists no $B_{c}$ or homoclinic orbits. For antiferromagnetic interactions, the analogous radius $r_{B}$ decreases from $r_{I}=\sqrt{2 \rho_{0}(0)\left[\left(1-\rho_{0}(0)\right)-\sqrt{\left(1-\rho_{0}(0)\right)^{2}-m^{2}}\right]}$ to zero while $B$ increases from zero to $B_{c}$. At $B=B_{c}$ the attractor-like feature remains. When $B$ is increased from $B_{c}, r_{B}$ increases from zero, and crosses $r_{I}$ at $B=B_{0}$, finally approaches the radius of the allowed region for a non-interacting condensate when $B \rightarrow \infty$.

Before concluding, we hope to make some estimates to support the use of the mean field theory, i.e. treating the atomic field operators as c-numbers. Intuitively, we would expect that this is a reasonable approximation as the total numbers of atom, at 1000, although not macro- 
scopic, is definitely 'large'. In fact, the recent double well experiment that confirmed the coherent nonlinear Josephson oscillations of the mean field theory, is at a similar level of numbers of atoms 21]. A rigorous discussion of this point in terms of the quantum phase diffusions in a spin- 1 condensate is a rather involved procedure, and will not be reproduced here [30]. Instead, we illuminate the validity of mean field theory as follows. First, we look at the total atom number fluctuations. Approximating the spinor condensate as a one component scalar, and neglecting the internal spin mixing dynamics, its total overall phase spreads after a time of $\tau_{c} \approx N /\left[\sigma(N)\left(c_{0}\langle n\rangle\right)\right]$ 31], with $\sigma(N) \sim \sqrt{N}$ the standard deviation of the atom numbers from taking c-number approximations of the atomic field operators. In our case, this time is about 0.2 second, short compared to a typical Josephson type oscillation period at $\sim 1$ second. We believe, however, this is not a critical issue as we are not studying phase sensitive phenomena involving the overall phase as in an interference experiment. Instead, we are interested here in the relative phase dynamics between different condensate components, whose oscillation time scale is given by the much smaller value of the spin-dependent interaction coefficient $c_{2}$; thus we should compare the coherent classical oscillation period of $\sim 1$ second with the much longer time $\tau_{c}^{\prime} \approx N /\left[\sigma(N)\left(c_{2}\langle n\rangle\right)\right]$ [17], $\sim 50$ seconds (for ${ }^{87} \mathrm{Rb}$ ). This then leads to a favorable condition for adopting the mean field theory in our study. Alter- natively, we can reach the same conclusion from a direct investigation of the oscillation period $T$ Eq. (8a), which contains a simple $N$ dependence $\propto 1 / \sqrt{N}$. We find that $|T(N \pm \sqrt{N})-T(N)| / T(N)=1 /(2 \sqrt{N})$, is only about $2 \%$, indicating the overall validity of the mean field theory.

In conclusion we have studied the off-equilibrium interaction driven collective oscillations inside an atomic condensate in an external uniform magnetic field. The dynamics of spin mixing is found to be well described by a nonrigid pendulum due to the conservation of atom numbers and atomic magnetization. In particular, we find that there exists an interesting class of critical trajectories whose oscillation periods approach infinity. Our study illuminates the use of quadratic Zeeman shift to probe pendulum-like oscillations in a spin- 1 condensate and provides the complete spin mixing dynamics analytically. It provides the much needed theoretical guidance for the eventual experimental detection of coherent macroscopic oscillations in a spinor condensate.

Note added in proof: We have recently observed many of the coherent oscillatory behavior discussed in this work and have submitted a publication describing these experiments.

W. Zhang thanks Dr. Su Yi for several discussions of this project. This work is supported by NSF and NASA.
[1] C. A. Regal, M. Greiner, and D. S. Jin, Phys. Rev. Lett. 92, 040403 (2004).

[2] M. W. Zwierlein, C. A. Stan, C. H. Schunck, S. M. F. Raupach, A. J. Kerman, and W. Ketterle, Phys. Rev. Lett. 92, 120403 (2004).

[3] M. R. Matthews, B. P. Anderson, P. C. Haljan, D. S. Hall, C. E. Wieman, and E. A. Cornell, Phys. Rev. Lett. 83, 2498 (1999).

[4] K. W. Madison, F. Chevy, W. Wohlleben, and J. Dalibard, Phys. Rev. Lett. 84, 806 (2000).

[5] C. Raman, J. R. Abo-Shaeer, J. M. Vogels, K. Xu, and W. Ketterle, Phys. Rev. Lett. 87, 210402 (2001).

[6] M. Greiner, O. Mandel, T. Esslinger, T. W. Hänsch, and I. Bloch, Nature (London) 415, 39 (2002).

[7] M. Olshanii, Phys. Rev. Lett. 81, 938 (1998).

[8] A. Görlitz, J. M. Vogels, A. E. Leanhardt, C. Raman, T. L. Gustavson, J. R. Abo-Shaeer, A. P. Chikkatur, S. Gupta, S. Inouye, T. Rosenband, and W. Ketterle, Phys. Rev. Lett. 87, 130402 (2001).

[9] J. Stenger, S. Inouye, D. M. Stamper-Kurn, H. -J. Miesner, A. P. Chikkatur, and W. Ketterle, Nature (London) 396, 345 (1998).

[10] D. M. Stamper-Kurn, M. R. Andrews, A. P. Chikkatur, S. Inouye, H. -J. Miesner, J. Stenger, and W. Ketterle, Phys. Rev. Lett. 80, 2027 (1998).

[11] M. D. Barrett, J. A. Sauer, and M. S. Chapman, Phys. Rev. Lett. 87, 010404 (2001).

[12] H. Schmaljohann, M. Erhard, J. Kronjäger, M. Kottke, S. van Staa, L. Cacciapuoti, J. J. Arlt, K. Bongs, and K.
Sengstock, Phys. Rev. Lett. 92, 040402 (2004).

[13] H. Schmaljohann, M. Erhard, J. Kronjäger, K. Bongs, and K. Sengstock, Appl. Phys. B 79, 1001 (2004).

[14] T. Kuwamoto, K. Araki, T. Eno, and T. Hirano, Phys. Rev. A 69, 063604 (2004).

[15] T. -L. Ho, Phys. Rev. Lett. 81, 742 (1998).

[16] T. Ohmi and K. Machida, J. Phys. Soc. Jpn. 67, 1822 (1998).

[17] C. K. Law, H. Pu, and N. P. Bigelow, Phys. Rev. Lett. 81, 5257 (1998).

[18] S. Yi, Ö. E. Müstecaplığlu, C. P. Sun, and L. You, Phys. Rev. A 66, 011601(R) (2002).

[19] H. Pu, C. K. Law, S. Raghavan, J. H. Eberly, and N. P. Bigelow, Phys. Rev. A 60, 1463 (1999).

[20] H. Pu, S. Raghavan, and N. P. Bigelow, Phys. Rev. A 61, 023602 (2000).

[21] M. Albiez, R. Gati, J. Fölling, S. Hunsmann, M. Cristiani, and M. K. Oberthaler, e-print cond-mat/0411757 v2.

[22] M. R. Matthews, B. P. Anderson, P. C. Haljan, D. S. Hall, M. J. Holland, J. E. Williams, C. E. Wieman, and E. A. Cornell, Phys. Rev. Lett. 83, 3358 (1999).

[23] J. Williams, R. Walser, J. Cooper, E. A. Cornell, and M. Holland, Phys. Rev. A 61, 033612 (2000).

[24] M. -S. Chang, C. D. Hamley, M. D. Barrett, J. A. Sauer, K. M. Fortier, W. Zhang, L. You, and M. S. Chapman, Phys. Rev. Lett. 92, 140403 (2004).

[25] W. Zhang, S. Yi, and L. You, New J. Phys. 5, 77 (2003).

[26] The parameters are taken from M. -S. Chang and M. S. 
Chapman's new experiment set up (different from [24]).

[27] J. Vanier and C. Audoin, The quantum physics of atomic frequency standards (A. Hilger, Philadelphia, 1988).

[28] D. R. Romano and E. J. V. de Passos, Phys. Rev. A 70, 043614 (2004).

[29] J. M. Higbie, L. E. Sadler, S. Inouye, A. P. Chikkatur, S. R. Leslie, K. L. Moore, V. Savalli, and D. M. Stamper-
Kurn, e-print cond-mat/0502517

[30] S. Yi, Ö. E. Müstecaplığlu, and L. You, Phys. Rev. Lett. 90, 140404 (2003).

[31] A. Imamog̃lu, M. Lewenstein, and L. You, Phys. Rev. Lett. 78, 2511 (1997). 